International Journal of Modern Physics D

(C) World Scientific Publishing Company

\title{
DISK-JET CONNECTION IN AGNS AND MICROQUASARS: THE POSSIBILITY OF THERMAL FLARES IN THE CENTER
}

\author{
JONI TAMMI* \& TALVIKKI HOVATTA ${ }^{\dagger}$ \\ Aalto University Metsähovi Radio Observatory, \\ Metsähovintie 114, FI-02540 Kylmälä, Finland
}

Received 10 December 2009

Revised 1 February 2010

\begin{abstract}
We discuss the possibility of thermal flares in centers of AGNs and microquasars. We present preliminary results of an ongoing study trying to assess the feasibility of a hypothesis suggesting that certain flares observed in these sources originate in the very centers of the systems and not in the relativistic jets. Using a simple toy model we reproduce optical flares with lightcurves very similar to those observed in the sources. The model suits especially well those cases where only the latter peak of a double-peaked optical flare has a radio counterpart.
\end{abstract}

Keywords: Active Galactic Nuclei; Microquasars; Accretion Disk; Radiation outburst.

\section{Introduction}

Many characteristics of flaring in active galactic nuclei (AGN) and microquasars are best explained with relativistic motion of the radiating regions in jets. Especially for microquasars the variability is often best explained by variations in the structure of the accretion disk and the jets; as discussed by many authors throughout this volume, the connection between the disk and the large-scale jets is well established even if the details are not yet fully known.

Our starting point for this report is the general model proposed for both the microquasars and AGNs (see refs. 1, 2, and references therein). The connection between the accretion disk and the radio jet in AGNs has been discussed from different angles, e.g., in refs. 22 6, and in general the scenario is believed to work along the following lines: part of the accretion disk breaks off, and while the inner hot X-ray emitting matter falls beyond the event horizon (causing a decrease in the $\mathrm{X}$-ray flux (see ref. 2 and references therein) the outer parts get injected into the jet that is collimated and accelerated before becoming visible at what is called the core of the jet. There the jet plasma is believed to be compressed and heated by a

\footnotetext{
*Email: joni.tammi@iki.fi

${ }^{\dagger}$ Current address: Department of Physics, Purdue University, 525 Northwestern Avenue, West Lafayette, IN 47907, USA. Email: thovatta@purdue.edu
} 
standing shock wave $\frac{314}{4}$ causing a multifrequency flare and a new knot becoming visible in the jet!5 Apart from the jet core this is the same process that was proposed for the microquasar GRS $1915+105$ already earlier. 1 In this report we suggest an additional component to the scenario.

Our study was motivated by the observations and idea of Miller-Jones et al., 7 who found that certain double-peaked flares in the Cygnus X-3 are best explained by treating the first flare as a product of an outbreak of disk wind, later followed by a second flare when a new jet element becomes visible further away in the jet. Here we study the possibility that this model is at work also in AGNs and that - in addition to getting swallowed by the black hole or being injected into the jet - part of the collapsing accretion disk matter erupts from the center as a windlike outflow. We test if this outburst of matter - originally having temperatures comparable to the inner parts of the disk - could produce observable effects or, in the most radical case, cause a flare comparable to those happening in the jet, thus leading to double-peaked flare. Modeling the causes of such an event is beyond the scope of this early-phase report - at this point we focus on estimating what observational signatures a sudden outflow of matter in the center of an AGN or microquasar could produce.

In the following we describe our hypothesis for the first flare in the center (Sec. 2) and discuss the expected signatures and, in Sec. 3, discuss possible predictions of the model concentrating on the application to AGNs. An example is given in Sec. 4 using BL Lacertae as a test case.

\section{Toy model for a thermal outburst in the center}

In this early phase of the study we use a very simplified model to see if a thermal flare with reasonable parameters can, even in principle, cause any of the observed features. For this test we take a "spherical cow" approach: we describe the radiating plasma as a homogeneous sphere expanding at a constant speed and compute the blackbody and the bremsstrahlung emission (taking the free-free absorption and the light-travel time effects into account) at different frequencies as a function of time.

For simplicity we take the matter to consist only of electrons and positrons initially having a temperature comparable to that of the accretion disk $\left(T_{0} \sim 10^{4-6}\right.$ $\mathrm{K}$ for AGN ${ }^{8}$ ), and calculate the matter density from the initial radius of the sphere $\left(r_{S}<R_{0} \lesssim 3 r_{\mathrm{S}}\right.$, where $r_{\mathrm{S}}=2 G M / c^{2}$ is the Schwartzschild radius of the black hole) and the mass of the collapsing disk, estimated to be of the order of 1-10 $M_{\odot}$, assuming the disk to contain mass accreted since the last major burst -typically occurring once every couple of years $\frac{9}{1}$ at a rate of a few $M_{\odot} / \mathrm{yr}$.

In the present model the sphere of plasma immediately starts to expand with a constant speed -which we assume to be mildly relativistic, up to $0.5 \mathrm{c}$ (see, e.g., ref. 10) - and also cool down due to adiabatic losses. 


\section{Double flares - observational features and predictions}

Different flare characteristics. As the mechanisms and the environments related to the first flare in the center and the second flare at the core of the jet are different, also the flares should differ from each other from spectral as well as temporal points of view. Firstly, with typical AGN parameters the sphere is optically thin for optical photons from the beginning, making the flare bright in the optical waveband almost immediately. Millimeter and radio flux, on the other hand, peak weeks to months later and reach flux levels of only small fractions of those of the optical peak. It is also important to notice that the timescales or radiative signatures of first flare would not be affected by similar Lorentz boosts or Doppler shifts than the flares in the jet.

Single or double flares, depending on frequency. Whereas a thermal flare would most likely be observed and interpreted as an optical flare without radio counterpart, a later flare (at the radio core) is always expected to be bright throughout the spectrum. This means that depending on the observing frequency, the two flares would be observed either as a single- or double-peaked flares (at the radio and optical wavelenghts, correspondingly).

Location of the core and delay between the flares. The delay between the two flares, $\Delta t$, would depend on the distance of the jet core from the central black hole, on the speed (and acceleration) of the jet, and on the light-travel-time effects. Assuming, for simplicity, instant acceleration of the jet to the speed corresponding to the apparent velocity $v_{\text {app }}$ at which the knots in the post-core jet (making an angle $\theta$ with the line of sight) are seen to travel, the separation of the core is simply $r_{\text {core }}=\Delta t v_{\text {app }} / \sin \theta$. Taking into account the finite time required for the acceleration of the jet, this may overestimate the actual distance.

Dip in the degree of polarization. Because the radiation from the first thermal flare is taken to be unpolarized, and if the intensity of polarized emission is not changed, the rise of the unpolarized radiation leads to a corresponding decrease of the polarization. As a zeroth-order estimate one can estimate the dip in the polarization simply from $P=P_{0} /\left(1+I_{\text {flare }}\right)$, where $P_{0}$ and $I_{\text {flare }}=\Delta I / I_{0}$ are the pre-flare polarization and the brightness increase compared to the pre-flare level.

Flux levels start to rise before the second flare. If the first flare loads the jet with matter, this could lead to the flux starting to rise already before the second flare. On one hand this is due to the particles in the plasma being energized by the strong magnetic turbulence near the center providing promising conditions for efficient second-order Fermi acceleration, 11 leading to increased synchrotron emissivity. On the other hand, the acceleration of the "proto jet" emitting region as a whole leads to increased Lorentz boosting of the emitted radiation. 


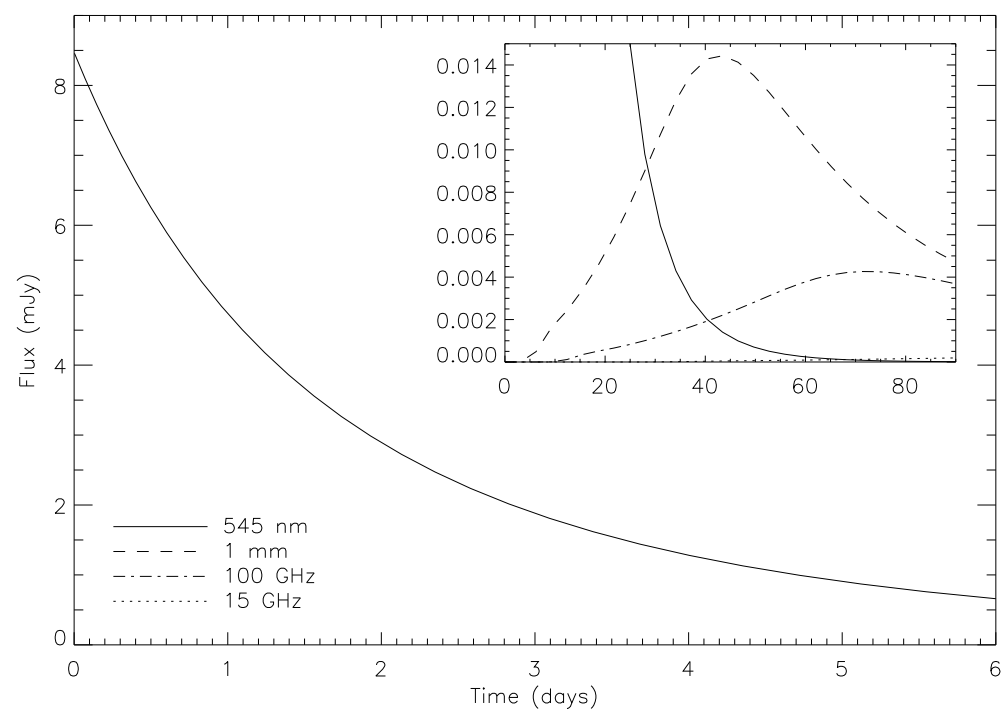

Fig. 1. Example lightcurves from a thermal flare in BL Lacertae for optical (solid line) and three radio frequencies as given in the plot. The dimmer and slower radio flares are only visible in the small panel showing the micro-Jansky level peaks months after the optical mJy-level flare. See text for details.

New "knot" in the jet after the second flare. Finally, when the jet-injected matter reaches the location of the VLBI core of the radio jet, the standing shock (assumed to lie in the core) compresses the plasma and accelerates particles on short timescales, causing a multifrequency flare. After passing through the core, the radiating plasma continues to travel along the jet and is now seen as a bright knot 516 From this point on the ejected plasma can be described using various shock-in-jet models.

\section{An example: BL Lacertae}

Let us illustrate the points raised in Sec. 3 in the case of the blazar BL Lac $(z=$ $0.069, M \approx 10^{8} M_{\odot}$ ). By assuming the outbursting mass to be $M_{\text {in }}=10 M_{\odot}$ (the major outbursts in the source happening on average every $2-3$ years, $\frac{12}{12}$ leading to required mass accretion rate, $3-5 M_{\odot} / \mathrm{yr}$ ), the expansion speed $v_{\exp }=0.4 \mathrm{c}$, initial radius $r_{0}=1.5 r_{\mathrm{S}}$, initial temperature $T_{0}=10^{5} \mathrm{~K}$ and adiabatic index $\gamma=4 / 3$, we get an optical flare rising very fast (within hours) to $8.5 \mathrm{mJy}$ and declining in a few days with $S \propto e^{-t / 2}$, approximately, as shown in Fig. 1 At the radio frequencies, however, the source becomes optically thin much later and has a much lower peak flux density keeping the radio signature of the flare invisible under the strong and variable nonthermal flux from the jet. In essence this would be seen as an optical flare without a radio counterpart.

To have even an order-of-magnitude reality check before detailed data analysis, 
we compare this to observations of BL Lac made with with the Kanata telescope in October 2008 Beginning on 11th of October 2008, we see an optical flare rising from $8.4 \mathrm{mJy}$ to $17.0 \mathrm{mJy}$ within a few days, the flux increasing by approximately 8.6 mJy. This is remarkably close to the $8.5 \mathrm{mJy}$ obtained from our zeroth-order model using first-guess input parameters. For the duration of the flare the polarization degree decreased from $18 \%$ to $11 \%$. Using the same initial polarization, we obtain the value of $9 \%$, still interestingly near the observed one. The comparison is, of course, very crude, but it is sufficient to keep the proposed scenario plausible.

\section{Discussion and Conclusions}

In addition to the presented case for BL Lac, we have tested our toy model for different AGNs and microquasars. The results (in preparation) are promising: even with our very crude spherical-cow model we have been able to reproduce lightcurves similar to those observed both in AGN and microquasar objects. Some sources, however, seem to be completely beyond the scope of the flux levels and timescales obtainable with the present simple model. We are currently collecting multifrequency data for detailed testing and further development.

Further developments of the model will enable testing the X-ray emission (enabling comparison with the observed anticorrelation between the X-ray and radio fluxes ${ }^{4}$ ). Also the possible role of nonthermal radiation, even in the case of a mostly thermal flare, needs to be studied. Furthermore, the physicality of the model will be improved by including different geometries and dynamics for the outflow, as well as taking into account the presence of, e.g., the dust torus for AGNs and companion star in microquasar, as well as the differences in the density and composition of the environment and the matter in the coronae of the black hole and the accretion disk.

Finally we comment on two recent reports that are relevant to the points presented in Sec. 3. Firstly, Marscher et al. ${ }^{[3}$ reported an example of an optical double peak with a single radio burst followed by a jet element in the jet of BL Lacertae. The first flare was shown to happen in the acceleration and collimation region before the radio core of the jet, following an explosive event near the black hole; they explain the first optical flare as a product of the increased radiation from the accelerating plasma blob before the core. In our model the flare would take place closer to the black hole - otherwise our model assumes everything to happen as described by Marscher et al ${ }^{3}$ The published data are, however, still too sparse to either support or disprove our model; further continuous and dense multifrequency observations are needed to distinguish the possible non-jet flares from those happening in the outflows or in the accretion disk. Secondly, a very recent article by Villforth et al 13 published polarization data of the BL Lac object OJ287. Their lightcurves included many peaks consisting mostly of unpolarized flares. In this source the variability is considered to be linked to the dynamics of a binary black hole with the secondary

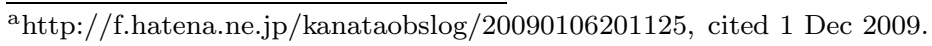


black hole disrupting the accretion disk (see their paper for a review of different models suggested for the source). Their results could provide interesting and very useful tool in testing the proposed model, as they emphasize the repeated doublepeaked outbursts with the first peak having no radio counterpart. Furthermore, they report dips in the optical polarization during the burst. We acknowledge, however, that the toy model presented here does not seem to be able to reproduce the brightness of OJ287 flares. We dare not yet speculate whether improved modeling of the black hole's environment could help, or can this kind of an approach apply even in theory in an object suspected to harbor a binary supermassive black hole (ref. 13 and references therein).

To conclude, based on the preliminary results and within the limits of currently available data we cannot rule out the possibility that in some microquasars and AGNs certain flares can be due to a thermal or partly thermal flare associated with the explosive event that also dismisses parts of the accretion disk and injects material into the jets. However, more data and improvements for the model are needed to satisfyingly estimate the feasibility of non-jet flares in these sources.

\section{Acknowledgments}

We thank the Kanata telescope observation blog for their openness in providing example data. Furthermore, Dr. Tuomas Savolainen is acknowledged for pointing out the possibility for using dip of the polarization degree in testing the model.

\section{References}

1. Mirabel, I. F. \& Rodríguez, L.F. 1998, Nature, 392, 673

2. Marscher, A.P., Jorstad, S.G., Gómez, J.-L., Aller, M.F., Teräsranta, H., Lister, M.L. \& Stirling, A.M. 2002, Nature, 417, 625

3. Marscher, A.P., Jorstad, S.G., D’Arcangelo, F.D., et al. 2008, Nature, 452, 966

4. Chatterjee, R., Marscher, A.P., Jorstad, S.G., et al. 2009, ApJ, 704, 1689

5. Savolainen, T., Wiik, K., Valtaoja, E., Jorstad, S. \& Marscher, A.P. 2002, A\&A, 394, 851

6. Chatterjee, R., Jorstad, S.G., Marscher, A.P., et al. 2008, ApJ, 689, 79

7. Miller-Jones, J.C.A., Blundell, K.M., Duffy, P., Tammi, J., Podsiadlowski, P., Mioduszewski, A.J., Rupen, M.P. \& Trushkin, S., in preparation

8. Bonning, E.W., Cheng, L., Shields, G.A., Salviander, S. \& Gebhardt, K. 2007, ApJ, 659, 21

9. Hovatta, T., Tornikoski, M., Lainela, M., Lehto, H. J., Valtaoja, E., Torniainen, I., Aller, M. F. \& Aller, H. M. 2007, A\&A, 469, 899

10. Tombesi, F., Cappi, M., Yaqoob, T., Reeves, J. \& Palumbo, G.G.C., in proc. of "Accretion and ejection in AGNs", Como, Italy, June 2009, ASP Conf.Proc. (in press)

11. Tammi, J. \& Duffy, P., 2009, MNARS, 393, 1063

12. Nieppola, E., Hovatta, T.,Tornikoski, M., Valtaoja, E., Aller, M. F. \& Aller, H. M. 2009, AJ, 137, 5022

13. Villforth, C., Nilsson, K., Heidt, J., et al., MNRAS (in press), pre-print: arXiv:0912.0005 v2 [astro-ph.CO] 